\title{
Noninvasive Assessment of Intracranial Pressure Status in Idiopathic Intracranial Hypertension Using Displacement Encoding with Stimulated Echoes (DENSE) MRI: A Prospective Patient Study with Contemporaneous CSF Pressure Correlation
}

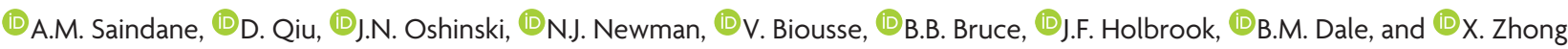

\section{ABSTRACT}

BACKGROUND AND PURPOSE: Intracranial pressure is estimated invasively by using lumbar puncture with CSF opening pressure measurement. This study evaluated displacement encoding with stimulated echoes (DENSE), an MR imaging technique highly sensitive to brain motion, as a noninvasive means of assessing intracranial pressure status.

MATERIALS AND METHODS: Nine patients with suspected elevated intracranial pressure and 9 healthy control subjects were included in this prospective study. Controls underwent DENSE MR imaging through the midsagittal brain. Patients underwent DENSE MR imaging followed immediately by lumbar puncture with opening pressure measurement, CSF removal, closing pressure measurement, and immediate repeat DENSE MR imaging. Phase-reconstructed images were processed producing displacement maps, and pontine displacement was calculated. Patient data were analyzed to determine the effects of measured pressure on pontine displacement. Patient and control data were analyzed to assess the effects of clinical status (pre-lumbar puncture, post-lumbar puncture, or control) on pontine displacement.

RESULTS: Patients demonstrated imaging findings suggesting chronically elevated intracranial pressure, whereas healthy control volunteers demonstrated no imaging abnormalities. All patients had elevated opening pressure (median, $36.0 \mathrm{~cm}$ water), decreased by the removal of CSF to a median closing pressure of $17.0 \mathrm{~cm}$ water. Patients pre-lumbar puncture had significantly smaller pontine displacement than they did post-lumbar puncture after CSF pressure reduction $(P=.001)$ and compared with controls $(P=.01)$. Post-lumbar puncture patients had statistically similar pontine displacements to controls. Measured CSF pressure in patients pre- and post-lumbar puncture correlated significantly with pontine displacement $(r=0.49 ; P=.04)$.

CONCLUSIONS: This study establishes a relationship between pontine displacement from DENSE MR imaging and measured pressure obtained contemporaneously by lumbar puncture, providing a method to noninvasively assess intracranial pressure status in idiopathic intracranial hypertension.

ABBREVIATIONS: DENSE = displacement encoding with stimulated echoes; ICP $=$ intracranial pressure; $I I H=$ idiopathic intracranial hypertension; IQR $=$ interquartile range; $L P=$ lumbar puncture

ntracranial pressure (ICP) reflects the pressure of the brain parenchyma and CSF. Many clinical disorders can abnormally elevate ICP through a variety of mechanisms, resulting in important clinical manifestations and findings on imaging. ${ }^{1}$ Idiopathic intracranial hypertension (IIH) is a condition of unknown etiol-

Received June 24, 2017; accepted after revision October 22.

From the Departments of Radiology and Imaging Sciences (A.M.S., D.Q., J.N.O. J.F.H.) and Ophthalmology (N.J.N., V.B., B.B.B.), Emory University, Atlanta, Georgia; Department of Biomedical Engineering (J.N.O.), Georgia Institute of Technology, Atlanta, Georgia; MR R\&D Collaborations (B.M.D.), Siemens Healthineers, Apex, North Carolina; and MR R\&D Collaborations (X.Z.), Siemens Healthineers, Atlanta, Georgia.

Please address correspondence to Amit M. Saindane, MD, Division of Neuroradiology, Emory University School of Medicine, Emory University Hospital, D112, 1364 Clifton Road, Atlanta, GA 30322; e-mail: asainda@emory.edu

http://dx.doi.org/10.3174/ajnr.A5486 ogy with elevated ICP unrelated to an intracranial mass lesion, meningeal process, or cerebral venous thrombosis., ${ }^{2,3}$ Patients with IIH are usually female, obese, and typically present with headaches, transient visual obscurations, and/or papilledema; treatment includes medication to decrease CSF production, weight loss, and/or CSF diversion. ${ }^{4}$ Measurement of ICP is essential for the diagnosis and management of IIH and many other neurologic and neurosurgical conditions and is typically estimated by lumbar puncture (LP) and CSF manometry. Because LP is invasive with small but definite risks and may be required at multiple time points for suspected treatment failure, it would be highly desirable to have a noninvasive and clinically practical technique to assess ICP status in IIH and other ICP disorders. $^{5}$

Displacement encoding with stimulated echoes (DENSE) is an MR imaging technique that encodes pixel-wise tissue displace- 
ments into the phase of the stimulated echoes. DENSE is wellsuited for measuring small displacements and has been shown to measure brain motion as small as $0.01 \mathrm{~mm}^{6}$ with good reproducibility. ${ }^{7}$ This study evaluates the ability of DENSE MR imaging to measure dynamic brain displacements in patients with IIH by using a tightly controlled protocol before and after LP and CSF removal with pressure reduction and compares these brain displacements to that of healthy control subjects. The hypothesis for this study is that high ICP constrains brain motion, but this brain motion can be normalized by the reduction of ICP through the removal of CSF. The validation of such a relationship would provide the basis for the use of DENSE brain displacement as a noninvasive means of assessing ICP status.

\section{MATERIALS AND METHODS}

\section{Patients and Control Subjects}

Approval for this prospective study was obtained from the Emory University institutional review board, and informed written consent was obtained from each participant. Nine patients, evaluated by a neuro-ophthalmologist between April 2015 and March 2017 at Emory University Hospital, with signs and symptoms of elevated ICP (eg, headaches, transient visual obscurations, and papilledema) were recruited and completed this study. Patients were excluded if they had standard contraindications to MR imaging or to gadolinium-based contrast agents. Exclusion criteria also included presence of an intracranial mass, hydrocephalus, or venous sinus thrombosis on MR imaging. Four additional suspected patients with IIH who were recruited and met the inclusion criteria were excluded from analysis because of excessive motion artifact on DENSE. Nine healthy nonobese volunteers were recruited as a comparison group. Although performance of LP on healthy volunteers was not considered ethically possible, each volunteer was questioned about history of headaches and visual disturbances.

\section{MRI Technique and Study Protocol}

All patients and healthy control subjects underwent MR imaging at 3T (Tim Trio; Siemens, Erlangen, Germany) in the supine position. A single midsagittal section through the brain was imaged by using a peripheral pulse unit-gated, segmented EPI, cine DENSE sequence. ${ }^{6}$ The pulse wave from the peripheral pulse unit was used for triggering the DENSE sequence. The image parameters were displacement encoding frequency $\left(\mathrm{k}_{\mathrm{e}}\right), 1.0$ or $1.5 \mathrm{cycle} / \mathrm{mm}$; through-plane dephasing frequency $\left(\mathrm{k}_{\mathrm{d}}\right), 0.08$ cycle/mm; TE, 8.9$10.4 \mathrm{~ms}$; TR, 55-59 ms; EPI factor, 8; segments, 16; pixel size, 1.2× $1.2 \mathrm{~mm}^{2}$; section thickness, $7 \mathrm{~mm}$; averages, 4; and frames, 10-20 (depending on the pulse duration). Different displacement frequencies were used in some subjects to acquire displacement information across a larger dynamic range. Displacement was encoded in the foot-to-head and anterior-to-posterior directions. The acquisition time for DENSE was approximately 2 minutes.

Control subjects underwent MPRAGE for planning and sagittal DENSE. Patients with IIH underwent the following tightly controlled protocol consisting of brain MR imaging and fluoroscopically guided LP: 1) The patient had MR imaging with sagittal T2 sampling perfection with application-optimized contrasts by using different flip angle evolution (SPACE; Siemens, Erlangen,
Germany), sagittal DENSE, axial DWI, axial T2*-weighted imaging, and axial T1-weighted imaging sequences; 2 ) The patient was then taken immediately to the fluoroscopy suite and an LP performed in the prone position with a 22 -gauge spinal needle and opening pressure measured by using CSF manometry; 3 ) CSF was withdrawn and the closing pressure measured; and 4) The patient was then taken immediately back for MR imaging with sagittal T2 SPACE, sagittal DENSE, contrast-enhanced MRV, axial fat-saturated T2-weighted imaging, axial postcontrast T1-weighted imaging, and sagittal postcontrast MPRAGE sequences.

\section{Image Analysis}

A subspecialty-certified neuroradiologist with 9 years of experience (A.M.S.) evaluated MR imaging and MRV images for intracranial abnormalities. DENSE images were exported for off-line processing in ImageJ (National Institutes of Health, Bethesda, Maryland). Phase-reconstructed images were divided by $2 \pi \mathrm{k}_{\mathrm{e}}$ to convert to displacement in millimeters. A single identical ROI was placed in the central pons on the magnitude images and then copied to the corresponding motion-encoded (foot-to-head) phase images (Fig 1A, -B). Mean displacement values in the ROIs were plotted over the phases of the cardiac cycle and the minimum value of displacement was subtracted from the maximum displacement to yield the maximum change in displacement across the cardiac cycle (Fig 1C). Postprocessing took 2-3 minutes per subject.

\section{Statistical Analysis}

Statistical analysis was performed by using SPSS version 22.0 (IBM, Armonk, New York). Patient and control demographics were compared by using the Fisher exact test and the Mann-Whitney $U$ test. Group differences in DENSE displacement between control subjects, pre-LP patients with IIH, and post-LP patients with IIH were evaluated by using a paired samples $t$ test for patients with IIH pre- and post-LP and an independent samples $t$ test for patients with IIH and control subjects. The relationship between measured pressure and DENSE displacement was assessed by using the Pearson correlation.

\section{RESULTS}

\section{Patients and Controls}

A total of 9 female patients with IIH were included in this study (Table 1). The median age for these patients was 28.1 years (interquartile range [IQR], 22.5-32.1 years), and the median body mass index was 38.9 (IQR, 28.1-41.8). All patients had an elevated opening pressure of $36.0 \mathrm{~cm}$ water (IQR, 32.5-41.0 cm water), a median of $15.0 \mathrm{~mL}$ CSF removed (IQR, 13-16 mL), and a resultant median closing pressure of $17.0 \mathrm{~cm}$ water (IQR, $15.5-19.0 \mathrm{~cm}$ water). The median change in pressure was $20.0 \mathrm{~cm}$ water (IQR, $15.0-23.0 \mathrm{~cm}$ water), representing a median $53 \%$ reduction of pressure (IQR, 47\%-58\%). The median time from pre-LP DENSE imaging to opening pressure measurement was $56.0 \mathrm{~min}$ utes (IQR, 40-72 minutes), and the median time from the closing pressure to the post-LP DENSE was 26.0 minutes (IQR, 17.5-34.0 minutes). None of the patients had unexpected structural intracranial abnormalities (eg, intracranial mass, hydrocephalus, ve- 

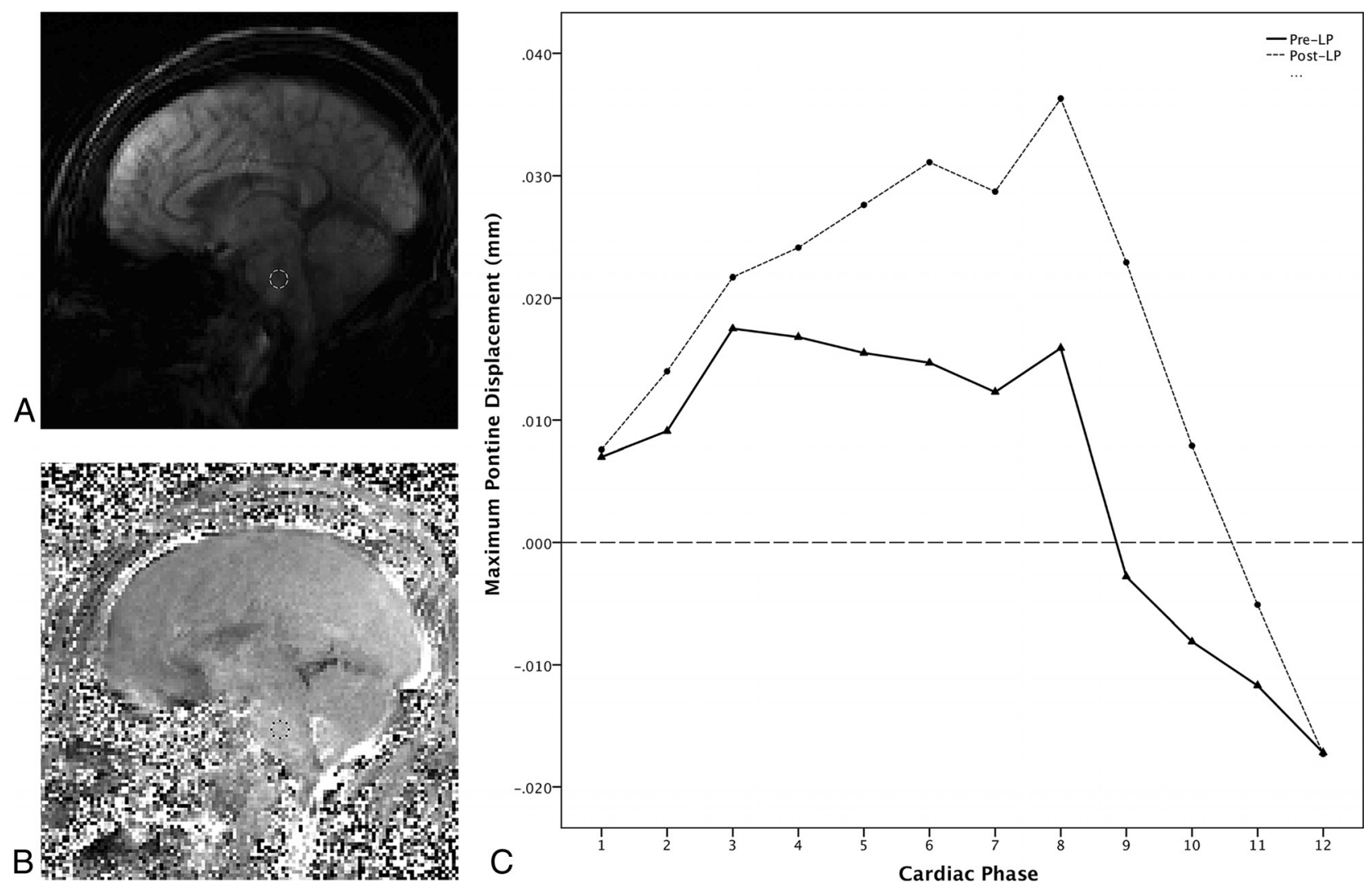

FIG 1. DENSE imaging with ROI placement and measured displacement across the cardiac cycle from a 44-year-old woman with IIH (Patient 9). $A$, Sagittal magnitude image from pre-LP DENSE encoded for motion in the foot-to-head direction. An ROI has been placed in the midpons to avoid partial volume effects from CSF flow. B. Corresponding phase image from the pre-LP DENSE encoded for motion in the foot-to-head direction with ROI transferred from the magnitude image into the midpons for measurement to be propagated to all 12 images acquired across the cardiac cycle. C, Graph showing DENSE displacement in the foot-to-head direction across the cardiac cycle divided into 12 phases. The solid line shows the pre-LP displacements across the cardiac cycle, and the dotted line shows post-LP displacements across the cardiac cycle. Maximum displacement is calculated by subtracting the lowest value from the highest across the cardiac cycle for both the pre-LP and post-LP states. Note the increased displacement in the post-LP state.

Table 1: Pressure measurements, DENSE pontine displacements, and times between measurements in patients with IIH pre-LP and post-LP

\begin{tabular}{|c|c|c|c|c|c|c|c|c|c|c|}
\hline $\begin{array}{c}\text { Patient } \\
\text { No. }\end{array}$ & 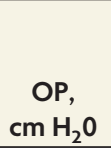 & 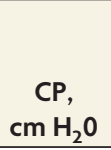 & $\begin{array}{c}\text { OP to } \mathrm{CP} \\
\text { Pressure } \\
\text { Change, } \mathrm{cm} \mathrm{H}_{2} \mathrm{O}\end{array}$ & $\begin{array}{l}\text { OP to } C P \\
\text { Pressure } \\
\text { Change, \% }\end{array}$ & $\begin{array}{c}\text { CSF } \\
\text { Removed, } \\
\text { cc } \\
\end{array}$ & $\begin{array}{c}\text { Pre-LP } \\
\text { DENSE, } \\
\mathrm{mm}\end{array}$ & $\begin{array}{l}\text { Post-LP } \\
\text { DENSE, } \\
\mathrm{mm}\end{array}$ & $\begin{array}{c}\% \\
\text { Change }\end{array}$ & $\begin{array}{c}\text { Pre-LP } \\
\text { DENSE to OP } \\
\text { Time, min }\end{array}$ & $\begin{array}{c}\text { CP to } \\
\text { Post-LP } \\
\text { DENSE } \\
\text { Time, min }\end{array}$ \\
\hline 1 & 26 & 15 & 11 & -42 & 11 & 0.124 & 0.134 & 8 & 56 & 17 \\
\hline 2 & 41 & 18 & 23 & -56 & 16 & 0.022 & 0.059 & 164 & 95 & 48 \\
\hline 3 & 36 & 17 & 19 & -53 & 20 & 0.071 & 0.097 & 36 & 67 & 18 \\
\hline 4 & 46 & 19 & 27 & -59 & 22 & 0.047 & 0.087 & 86 & 204 & 35 \\
\hline 5 & 40 & 16 & 24 & -60 & 15 & 0.105 & 0.119 & 14 & 51 & 34 \\
\hline 6 & 33 & 16 & 17 & -52 & 15 & 0.037 & 0.051 & 39 & 39 & 21 \\
\hline 7 & 35 & 15 & 20 & -57 & 15 & 0.024 & 0.052 & 113 & 72 & 10 \\
\hline 8 & 32 & 19 & 13 & -41 & 14 & 0.078 & 0.085 & 8 & 41 & 30 \\
\hline 9 & 49 & 26 & 23 & -47 & 12 & 0.035 & 0.054 & 54 & 35 & 26 \\
\hline
\end{tabular}

Note:-CP indicates closing pressure; OP, opening pressure.

nous sinus thrombosis, etc) to explain elevated ICP, though previously described imaging findings associated with elevated ICP were found in all patients involving the orbits (distension of the perioptic nerve subarachnoid space, flattening of the posterior sclera, intraocular protrusion of the prelaminar optic nerve, enhancement of the prelaminar optic nerve, and vertical tortuosity of the orbital optic nerve), ${ }^{8}$ skull base ("empty" sella turcia and prominent Meckel caves), ${ }^{9,10}$ cerebellar tonsils, ${ }^{11}$ and venous sinuses (transverse venous sinus stenosis). ${ }^{12}$ All patients had papill- edema and met the formal diagnostic criteria for IIH. Seven of 9 patients are followed regularly by neuro-ophthalmology, received standard medical treatment including acetazolamide and weight loss, and have experienced no visual loss. Follow-up is not available for 2 patients to assess their clinical outcomes.

A total of 9 healthy control subjects were included in the study ( 7 men and 2 women). The median age for control subjects was 34.0 years (IQR, 23.5-40.0 years). No control subject reported a history of chronic headaches or visual disturbances. 


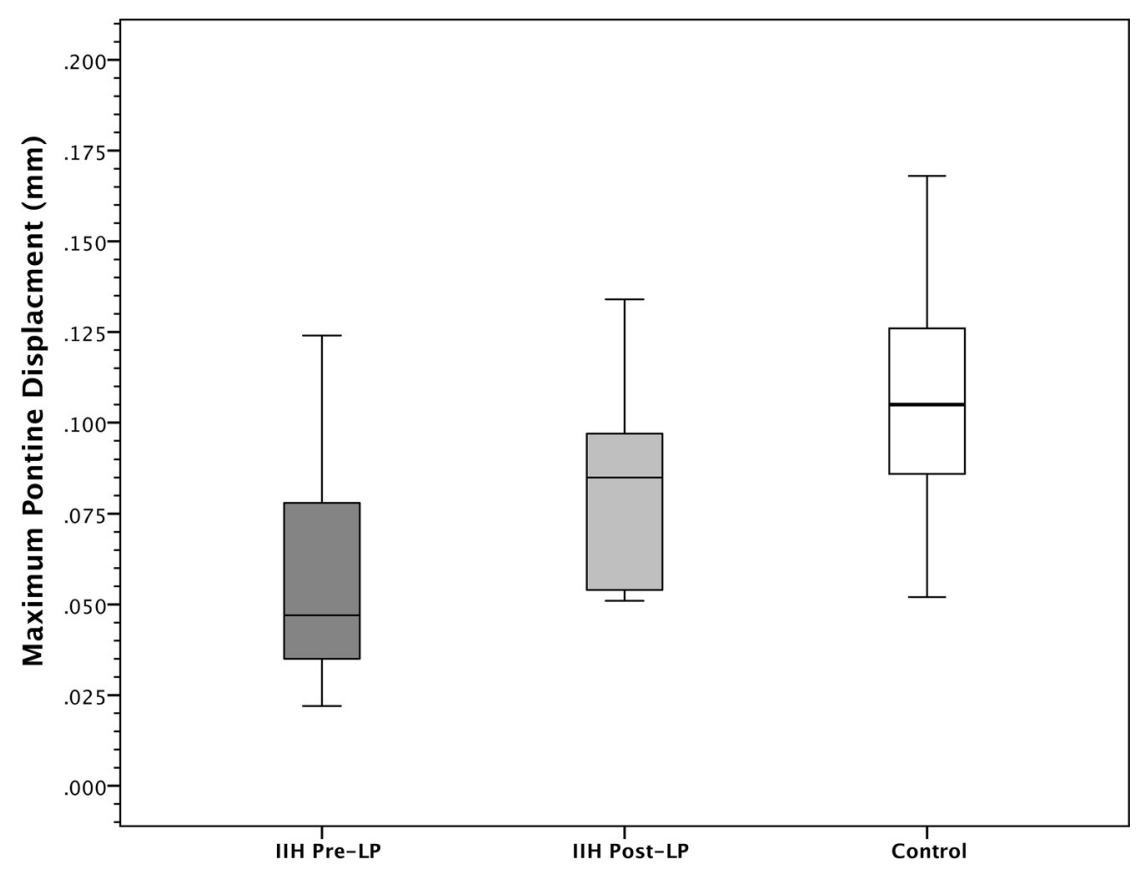

FIG 2. Comparison of maximum pontine displacement in pre-LP, post-LP, and control groups. The maximum pontine displacement of an ROI in the central pons in the pre-LP IIH group was significantly lower than in the post-LP state within subjects $(P<.001)$ and significantly lower than the control group $(P=.01)$. The maximum pontine displacement in post-LP patients with IIH did not significantly differ from control subjects $(P=.10)$. Values are shown in mean \pm SD.

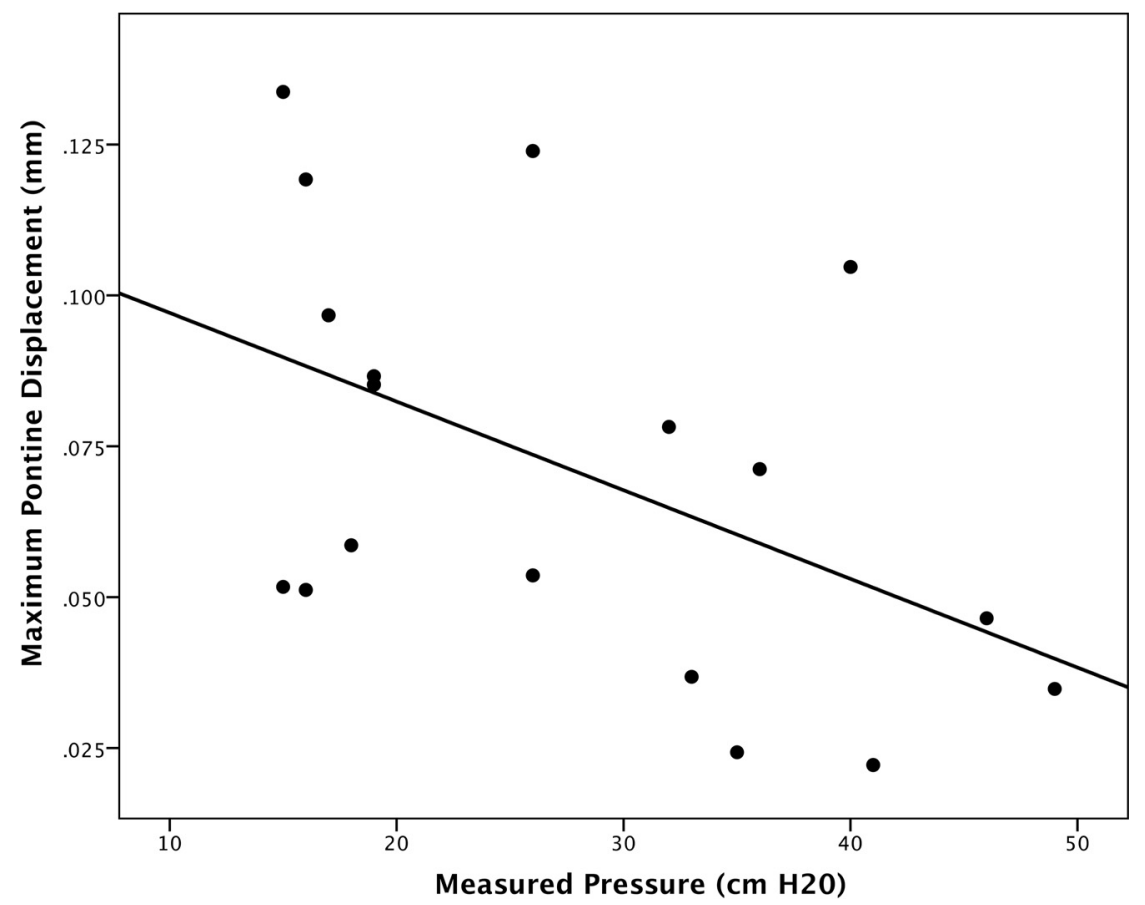

FIG 3. Correlation of measured CSF pressure and pontine displacement by DENSE. The maximum pontine displacement measured by DENSE correlates moderately with measured pressure (opening pressure or closing pressure) by CSF manometry $(r=0.49 ; P=.04)$.

All healthy control subjects had normal brain imaging, including no findings to suggest chronically elevated ICP. Patients and healthy control subjects did not significantly differ in age $(P=.35)$, though there were significant sex differences $(P=$ $.002)$.

\section{DENSE Analysis}

For patients with IIH, the mean pre-LP pontine displacement was $0.060 \mathrm{~mm}$ $(\mathrm{SD}=0.036 \mathrm{~mm})$, which was increased to a mean of $0.082 \mathrm{~mm}(\mathrm{SD}=0.031$ $\mathrm{mm})$ post-LP, representing a range of $8 \%$ to $164 \%$ increase in pontine displacement (mean of $58 \% ; P=.001$ ). Control subjects demonstrated a mean pontine displacement of $0.109 \mathrm{~mm}$ $(\mathrm{SD}=0.034 \mathrm{~mm})$, which was significantly higher than the pre-LP patients with $\operatorname{IIH}(P=.01)$, but not significantly different from the post-LP patients with $\operatorname{IIH}(P=.10$; Fig 2$)$. Across patients using both pre-LP and post-LP measured CSF pressures and DENSE measurements, there was a significant moderate correlation between pressure and pontine displacement by using DENSE $(r=-0.49 ; P=.04 ;$ Fig 3$)$.

\section{DISCUSSION}

This study prospectively used a protocol that tightly coupled DENSE MR imaging with the CSF opening pressure and closing pressure measured by LP and CSF manometry. We chose IIH as a model for elevated ICP because there is no intracranial abnormality such as a mass lesion that would vary in size or effect between patients. We hypothesized that elevated ICP would constrain peak brain motion because vascular pulsations may be less able to drive brain motion in a high-pressure state. We specifically evaluated the foot-to-head direction of motion and the brain stem because this had previously been the direction and location of the highest brain motion in healthy volunteers ${ }^{6}$ (compared with the anteroposterior direction, presumably related to increased compliance of the spinal canal from the presence of neural foraminal openings and epidural fat). The midpons was chosen as an easily identifiable landmark for ROI placement where there would not be partial volume effects with CSF flow. In comparison with healthy control subjects, patients with IIH had significantly decreased pontine displacement. Reducing CSF pressure through CSF removal resulted in withinsubject increase in pontine displacement for all patients with $\mathrm{IIH}$, with mean post-LP pontine displacement similar to that of healthy control subjects. Establishing this relationship between pressure and pontine displacement by using DENSE raises the 
potential for its use for noninvasive assessment of ICP status. Merits to the technique include a very short acquisition time and simple postprocessing, which could easily be automated for inline real-time clinical use. Specifically, the technique could be used to noninvasively track patients after a baseline DENSE measurement to assess for therapy failure in IIH and potentially other conditions (eg, CSF shunt failure as a cause of recurrent headache).

The reference standard for ICP measurement is direct ICP monitoring, which is highly invasive and not practical or necessary for most patients because CSF pressure by LP generally agrees with ICP. ${ }^{13}$ Orbital, skull base, dural venous sinus, and other imaging findings ${ }^{8-12}$ have been described in $\mathrm{IIH}$, but are not of sufficient accuracy to be diagnostic of elevated $\mathrm{ICP}^{14,15}$ and may persist after pressure reduction. ${ }^{16}$ Clinical examination of papilledema by using the Frisén scale varies among medical specialists, ${ }^{17}$ and the absence of papilledema does not exclude ICP elevation. Transcranial Doppler sonography and optic nerve ultrasonography have shown variable results for the assessment of ICP. ${ }^{18,19}$ An MR imaging approach derived from transcranial CSF flow and blood volumetric flow rates has been used to differentiate between normal and elevated $\mathrm{ICP}^{20,21}$; however, this approach is complex for clinical use and requires further investigation in a larger cohort.

There are several limitations to this study, including the relatively small number of patients evaluated. We attempted to minimize time lags between pressure and DENSE measurements; however, transport and procedural time precluded tighter coupling of the steps. Because CSF is regenerated over time, it could increase pressure post-LP above the measured closing pressure, introducing systematic error into the post-LP DENSE. Variability in the timing interval between DENSE and pressure measurement across patients could introduce additional error. Patients and control subjects differed in demographic features, so it is difficult to exclude the contributions of these and other variables to the comparisons between IIH and control groups. Next, there is substantial variability between patients in baseline pontine displacement and in their extent of change after LP and CSF removal. A variety of factors, including the extent and duration of elevated ICP and variations in potential adaptive responses related to the elevated ICP, could be responsible for this finding; therefore, the degree of change in pontine displacement through CSF pressure reduction may vary by patient-specific factors. We did not use a head immobilization device or correct in postprocessing for any bulk head motion, and this would perhaps further improve the robustness of the results in terms of interpatient differences and differences pre- to post-LP. An LP was not performed on healthy control subjects, so their exact ICP status is not known; however, the selection of nonobese control subjects without self-reported symptoms of elevated ICP makes it extremely unlikely that they have undiagnosed IIH. Finally, it is unclear how DENSE would perform in the setting of an intracranial mass, hydrocephalus, or other important brain abnormality or in circumstances where there is only mild elevation of ICP. Future extension to a larger cohort with normal and abnormally elevated ICP, as well as to other scanner systems and patients with other etiologies of abnor- mal ICP, will be required to determine the broader applicability of the DENSE technique.

\section{CONCLUSIONS}

This study establishes a relationship between pontine displacement obtained by DENSE MR imaging and CSF pressure obtained contemporaneously by LP in patients with IIH, providing a potential method for noninvasively assessing ICP status.

Disclosures: Deqiang Qiu—UNRELATED: Grants/Grants Pending: Siemens Medical Solutions*. John N. Oshinski-UNRELATED: Grants/Grants Pending: Siemens Medical Solutions, Comments: we (Emory) received a grant on the development of DENSE MRI applications. The grant work did not directly relate to this project, but is on a similar topic*. Nancy J. Newman—UNRELATED: Consultancy: GenSight Biologics, Santhera Pharmaceuticals, Quark Pharmaceuticals, Comments: consultant for GenSight Biologics and Santhera Pharmaceuticals, Data Safety Monitoring Board for Quark; Expert Testimony: Medical-legal firms, Comments: medical-legal expert for law firms on topics of papilledema and idiopathic intracranial hypertension. Valérie Biousse-UNRELATED: Consultancy: GenSight Biologics. Beau B. BruceUNRELATED: Consultancy: Medlmmune, Comments: Data Safety Monitoring Board; Expert Testimony: Bayer, Comments: individual litigants; Grants/Grants Pending: National Institutes of Health*. Brian M. Dale-UNRELATED: Employment: Siemens Healthcare. Xiaodong Zhong-UNRELATED: Employment: Siemens Medical Solutions USA. *Money paid to the institution.

\section{REFERENCES}

1. Holbrook J, Saindane AM. Imaging of intracranial pressure disorders. Neurosurgery 2017;80:341-54 CrossRef Medline

2. Friedman DI, Jacobson DM. Diagnostic criteria for idiopathic intracranial hypertension. Neurology 2002;59:1492-95 CrossRef Medline

3. Friedman DI, Liu GT, Digre KB. Revised diagnostic criteria for the pseudotumor cerebri syndrome in adults and children. Neurology 2013;81:1159-65 CrossRef Medline

4. Friedman DI, Jacobson DM. Idiopathic intracranial hypertension. J Neuroophthalmol 2004;24:138-45 CrossRef Medline

5. Bruce BB. Noninvasive assessment of cerebrospinal fluid pressure. J Neuroophthalmol 2014;34:288-94 CrossRef Medline

6. Zhong X, Meyer CH, Schlesinger DJ, et al. Tracking brain motion during the cardiac cycle using spiral cine-DENSE MRI. Med Phys 2009;36:3413-19 CrossRef Medline

7. Soellinger M, Rutz AK, Kozerke S, et al. 3D cine displacement-encoded MRI of pulsatile brain motion. Magn Reson Med 2009;61: 153-62 CrossRef Medline

8. Brodsky MC, Vaphiades M. Magnetic resonance imaging in pseudotumor cerebri. Ophthalmology 1998;105:1686-93 CrossRef Medline

9. Saindane AM, Lim PP, Aiken A, et al. Factors determining the clinical significance of an "empty" sella turcica. AJR Am J Roentgenol 2013;200:1125-31 CrossRef Medline

10. Bialer OY, Rueda MP, Bruce BB, et al. Meningoceles in idiopathic intracranial hypertension. AJR Am J Roentgenol 2014;202:608-13 CrossRef Medline

11. Aiken AH, Hoots JA, Saindane AM, et al. Incidence of cerebellar tonsillar ectopia in idiopathic intracranial hypertension: a mimic of the Chiari I malformation. AJNR Am J Neuroradiol 2012;33: 1901-06 CrossRef Medline

12. Farb RI, Vanek I, Scott JN, et al. Idiopathic intracranial hypertension: the prevalence and morphology of sinovenous stenosis. Neurology 2003;60:1418-24 CrossRef Medline

13. Lenfeldt N, Koskinen LO, Bergenheim AT, et al. CSF pressure assessed by lumbar puncture agrees with intracranial pressure. $\mathrm{Neu}$ rology 2007;68:155-58 CrossRef Medline

14. Bidot S, Saindane AM, Peragallo JH, et al. Brain imaging in idiopathic intracranial hypertension. J Neuroophthalmol 2015;35:400-11 CrossRef Medline

15. Agid R, Farb RI. Neuroimaging in the diagnosis of idiopathic intracranial hypertension. Minerva Med 2006;97:365-70 Medline

16. Bono F, Giliberto C, Mastrandrea C, et al. Transverse sinus stenoses 
persist after normalization of the CSF pressure in IIH. Neurology 2005;65:1090-93 CrossRef Medline

17. Johnson LN, Hepler RS, Bartholomew MJ. Accuracy of papilledema and pseudopapilledema detection: a multispecialty study. J Fam Pract 1991;33:381-86 Medline

18. Ballantyne SA, O'Neill G, Hamilton R, et al. Observer variation in the sonographic measurement of optic nerve sheath diameter in normal adults. Eur J Ultrasound 2002;15:145-49 CrossRef Medline

19. Ragauskas A, Bartusis L, Piper I, et al. Improved diagnostic value of a TCD-based non-invasive ICP measurement method compared with the sonographic ONSD method for detecting elevated intracranial pressure. Neurol Res 2014;36:607-14 CrossRef Medline

20. Tain RW, Bagci AM, Lam BL, et al. Determination of cranio-spinal canal compliance distribution by MRI: methodology and early application in idiopathic intracranial hypertension. J Magn Reson Imaging 2011;34:1397-404 CrossRef Medline

21. Alperin NJ, Lee SH, Loth F, et al. MR-intracranial pressure (ICP): a method to measure intracranial elastance and pressure noninvasively by means of MR imaging: baboon and human study. Radiology 2000;217:877-85 CrossRef Medline 